\title{
Positioned numerical semigroups with maximal gender as function of multiplicity and Frobenius number
}

\author{
J. C. Rosales ${ }^{1}$ (D), M. B. Branco ${ }^{* 2}$ (D), M. C. Faria ${ }^{3}$ (D) \\ ${ }^{1}$ Departamento de Álgebra, Universidad de Granada, E-18071 Granada, Spain \\ ${ }^{2}$ Departamento de Matemática, Universidade de Évora, 7000 Évora, Portugal \\ ${ }^{3}$ Área Departamental de Física, Instituto Sup. Eng. de Lisboa, 1959-007 Lisboa, Portugal
}

\begin{abstract}
A $C$-semigroup (respectively a $D$-semigroup) is a positioned numerical semigroup $S$ such that $\mathrm{g}(S)=\frac{\mathrm{F}(S)+\mathrm{m}(S)-1}{2}$ (respectively $\mathrm{g}(S)=\frac{\mathrm{F}(S)+\mathrm{m}(S)-2}{2}$ ). In this paper we study these semigroups giving formulas for the Frobenius number, pseudo-Frobenius number, and type. Furthermore, we give algorithms for computing the whole set of $C$-semigroups and $D$-semigroups.
\end{abstract}

Mathematics Subject Classification (2020). 20M14, 11D07

Keywords. numerical semigroups, positioned numerical semigroups, $C$-semigroups, $D$-semigroups tree, Frobenius number, multiplicity and gender

\section{Introduction}

Let $\mathbb{Z}$ be the set of integers and $\mathbb{N}=\{x \in \mathbb{Z} \mid x \geq 0\}$. A numerical semigroup is a nonempty subset $\mathrm{S}$ of $\mathbb{N}$ that is closed under addition, contains the zero element, and whose complement in $\mathbb{N}$ is finite. Numerical semigroups appear in several areas of mathematics and there are several interesting combinatorial invariants of a semigroup (see for example [11]). Notable numerical semigroup invariants include the Frobenius number, multiplicity, and gender of $S$ that are $\mathrm{F}(S)=\max \{x \in \mathbb{Z} \mid x \notin S\}, \operatorname{m}(S)=\min (S \backslash\{0\})$, and $\mathrm{g}(S)=\operatorname{card}(\mathbb{N} \backslash S)$, respectively.

Given a rational number $q$, we denote by $\lfloor q\rfloor=\max \{z \in \mathbb{Z} \mid z \leq q\}$, and $\lceil q\rceil=$ $\min \{z \in \mathbb{Z} \mid z \geq q\}$.

Let $k$ be a positive integer. A numerical semigroup $S$ is $k$-positioned if for all $x \in \mathbb{N} \backslash S$ we have that $k-x \in S$. The $\mathrm{F}(S)$-positioned numerical semigroups are the symmetric numerical semigroups studied in [6], [1] and [7]. The $\mathrm{F}(S)+\mathrm{m}(S)$-positioned numerical semigroups (respectively $\mathrm{F}(S)+\mathrm{m}(S)+1$-positioned) called positioned numerical semigroups (respectively almost-positioned) are studied in [2] (respectively [3]). Thus a numerical semigroup $S$ is positioned if for all $x \in \mathbb{N} \backslash S$ we have that $\mathrm{F}(S)+\mathrm{m}(S)-x \in S$. In [2, Proposition 5] it is shown that if $S$ is a positioned numerical semigroup then

*Corresponding Author.

Email addresses: jrosales@ugr.es (J. C. Rosales), mbb@uevora.pt (M. B. Branco), mfaria@adf.isel.ipl.pt (M. C. Faria)

Received: 15.04.2021; Accepted: 14.09.2021 
$\left\lceil\frac{\mathrm{F}(S)+1}{2}\right\rceil \leq \mathrm{g}(S) \leq\left\lfloor\frac{\mathrm{F}(S)+\mathrm{m}(S)-1}{2}\right\rfloor$. The aim of this paper is to study the positioned numerical semigroups $S$ for which $\mathrm{g}(S)=\left\lfloor\frac{\mathrm{F}(S)+\mathrm{m}(S)-1}{2}\right\rfloor$. In order to study this we distinguish two cases depending on the parity of $\mathrm{F}(S)+\mathrm{m}(S)$. A $C$-semigroup (respectively a $D$-semigroup) is a positioned numerical semigroup $S$ such that $\mathrm{g}(S)=\frac{\mathrm{F}(S)+\mathrm{m}(S)-1}{2}$ (respectively $\mathrm{g}(S)=\frac{\mathrm{F}(S)+\mathrm{m}(S)-2}{2}$ ).

Let $\mathcal{A}$ be a nonempty subset of $\mathbb{N}$. We denote by $\langle\mathcal{A}\rangle$ the submonoid of $(\mathbb{N},+)$ generated by $\mathcal{A}$, that is,

$$
\langle\mathcal{A}\rangle=\left\{\sum_{i=1}^{n} \lambda_{i} a_{i} \mid n \in \mathbb{N} \backslash\{0\}, a_{1}, \ldots, a_{n} \in \mathcal{A}, \text { and } \lambda_{1}, \ldots, \lambda_{n} \in \mathbb{N}\right\} .
$$

It is well known (see for example [11]) that $\langle\mathcal{A}\rangle$ is a numerical semigroup if and only if $\operatorname{gcd}(\mathcal{A})=1$.

If $S$ is a numerical semigroup and $S=\langle\mathcal{A}\rangle$ then we say that $\mathcal{A}$ is a system of generators of $S$. Moreover, if $S \neq\langle\mathcal{B}\rangle$ for all $\mathcal{B} \varsubsetneqq \mathcal{A}$, then we say that $\mathcal{A}$ is a minimal system of generators of $S$. In [11] it is proved that every numerical semigroup $S$ admits a unique minimal system of generators and its cardinality is upper bounded by $\mathrm{m}(S)$. We denote by $\operatorname{msg}(S)$ the minimal system generators of $S$. Its cardinality is the embedding dimension of $S$, denoted by e $(S)$.

A numerical semigroup is irreducible if it cannot be expressed as the intersection of two numerical semigroups properly containing it. In [10] it is shown that a numerical semigroup is irreducible if and only if it is either a symmetric or a pseudo-symmetric numerical semigroup. This class of semigroups is quite interesting in numerical semigroup theory (see for instance [6], [1], [5]) and there are numerous characterizations for it.

Proposition 1.1. [11, Proposition 4.4] Let $S$ be a numerical semigroup. $S$ is symmetric (resp. pseudo-symmetric) if and only if $\mathrm{F}(S)$ is odd (resp. even) and $x \in \mathbb{Z} \backslash S$ implies $\mathrm{F}(S)-x \in S$ (resp. $x \in \mathbb{Z} \backslash S$ implies that either $\mathrm{F}(S)-x \in S$ or $x=\frac{\mathrm{F}(S)}{2}$ ).

Given a numerical semigroup $S$, we say that an integer $x$ is a pseudo-Frobenius number if $x \in \mathbb{Z} \backslash S$ and $x+s \in S$ for all $s \in S \backslash\{0\}$. We denote by $\operatorname{PF}(S)$ the set of pseudo-Frobenius numbers of $S$, and its cardinality is the type of $\mathrm{S}$, denoted by $\mathrm{t}(S)$.

In section 2 we show that $S$ is a $C$-semigroup if and only if $S=S^{\prime} \cup\left\{\mathrm{F}\left(S^{\prime}\right)\right\}$ with $S^{\prime}$ a symmetric numerical semigroup different from $\mathbb{N}$ and $\langle\{2,3\}\rangle$. We will also give formulas for Frobenius number, pseudo-Frobenius numbers and type of a $C$-semigroup in terms of its minimal system of generators.

Let $S$ be a numerical semigroup and $\operatorname{msg}(S)=\left\{n_{1}, \ldots, n_{p}\right\}$. We say that an element $s \in S$ has unique expression if there exists a unique $n$-tuple $\left(\lambda_{1}, \ldots, \lambda_{p}\right) \in \mathbb{N}^{p}$ such that $s=\lambda_{1} n_{1}+\cdots+\lambda_{p} n_{p}$.

In section 3 we see that $S$ is a $D$-semigroup if and only if $\frac{\mathrm{F}(S)+\mathrm{m}(S)}{2} \in \operatorname{msg}(S)$ and $\mathrm{F}(S)+\mathrm{m}(S)$ has unique expression in $S$. The same way, as before, we show that $S$ is a $D$-semigroup if and only if $S=S^{\prime} \cup\left\{\mathrm{F}\left(S^{\prime}\right), \frac{\mathrm{F}\left(S^{\prime}\right)}{2}\right\}$ with $S^{\prime}$ a pseudo-symmetric numerical semigroup with $\mathrm{F}\left(S^{\prime}\right)>2 \mathrm{~m}\left(S^{\prime}\right)$. Again, we will also give formulas for the Frobenius number, pseudo-Frobenius numbers and type of a $D$-semigroup in terms of its minimal system of generators.

Finally, in section 4, the study done in the previous sections along with [4] allows us to give a procedure to compute the whole set of $C$-semigroups and $D$-semigroups with given Frobenius number and multiplicity.

\section{2. $C$-semigroups}

Recall that a $C$-semigroup is a positioned numerical semigroup $S$ with $\mathrm{g}(S)=\frac{\mathrm{F}(S)+\mathrm{m}(S)-1}{2}$. Our aim in this section is to characterize this type of numerical semigroups. 
A numerical semigroup $\{0, \mathrm{~m}(S) \rightarrow\}$ is sometimes called in the literature half-line or ordinary and it will be denoted here by $\Delta(m)$.

Proposition 2.1. If $m \in \mathbb{N} \backslash\{0,1\}$, then $\Delta(m)$ is a $C$-semigroup.

Proof. The half-line $\Delta(m)$ is a positioned numerical semigroup with $\mathrm{m}(\Delta(m))=m$, $\mathrm{F}(\Delta(m))=m-1$ and $\mathrm{g}(\Delta(m))=m-1$. Then we deduce that $\mathrm{g}(\Delta(m))=\frac{\mathrm{F}(\Delta(m))+\mathrm{m}(\Delta(m))-1}{2}$ and thus $\Delta(m)$ is a $C$-semigroup.

Given a numerical semigroup $S$, denote by

$$
Q(S)=\{x \in S \mid 1 \leq x \leq \mathrm{F}(S)+\mathrm{m}(S)-1\} .
$$

Its cardinality is denoted by $\mathrm{q}(S)$.

It is easy to prove our next result which is deduced in [2, Proposition 5].

Lemma 2.2. Let $S$ be a positioned numerical semigroup. Then

(1) $\phi: \mathbb{N} \backslash S \rightarrow Q(S)$, defined by $\phi(x)=\mathrm{F}(S)+\mathrm{m}(S)-x$ is an injective map.

(2) $\mathrm{g}(S) \leq \mathrm{q}(S)$.

(3) $S$ is a $C$-semigroup if and only if $\mathrm{g}(S)=\mathrm{q}(S)$.

Note that if $m \in \operatorname{msg}(S)$ and $x \in S \backslash\{0, m\}$ then $m-x \notin S$ and so we have the following result.

Lemma 2.3. Let $S$ be a numerical semigroup and $m \in \operatorname{msg}(S)$. If $x \in\{1, \ldots, m-1\} \cap S$ then $m-x \in\{1, \ldots, m-1\} \cap \mathbb{N} \backslash S$.

From the previous lemma we can state the next result.

Lemma 2.4. Let $S$ be a numerical semigroup such that $\mathrm{F}(S)+\mathrm{m}(S) \in \operatorname{msg}(S)$. Then the following conditions hold:

(1) $\psi: Q(S) \rightarrow \mathbb{N} \backslash S$, defined by $\psi(x)=\mathrm{F}(S)+\mathrm{m}(S)-x$ is a injective map.

(2) $\mathrm{q}(S) \leq \mathrm{g}(S)$.

Theorem 2.5. Let $S$ be a positioned numerical semigroup. Then $S$ is a $C$-semigroup if and only if $\mathrm{F}(S)+\mathrm{m}(S) \in \operatorname{msg}(S)$.

Proof. Necessity. If $\mathrm{F}(S)+\mathrm{m}(S) \notin \operatorname{msg}(S)$, then there exists $\{x, y\} \subseteq S \backslash\{0\}$ such that $\mathrm{F}(S)+\mathrm{m}(S)=x+y$. By Lemma 2.2, we deduce that $\phi$ is not a surjective map and so $\mathrm{g}(S)<\mathrm{q}(S)$. By using again (3) of the same lemma, we have that $S$ is not a $C$-semigroup.

Sufficiency. By using Lemmas 2.2 and 2.4, we conclude that $\mathrm{g}(S) \leq \mathrm{q}(S)$ and $\mathrm{q}(S) \leq$ $\mathrm{g}(S)$. Hence, $\mathrm{g}(S)=\mathrm{q}(S)$ and by (3) of Lemma 2.2 we obtain that $S$ is a $C$-semigroup.

Given a numerical semigroup $S$, we denote by $M(S)=\max (\operatorname{msg}(S))$. It is easy to prove the following result.

Lemma 2.6. Let $S$ be a numerical semigroup. If $\mathrm{F}(S)+\mathrm{m}(S) \in \operatorname{msg}(S)$, then $M(S)=$ $\mathrm{F}(S)+\mathrm{m}(S)$.

The following result is well-known and appears in [11].

Lemma 2.7. Let $S$ be a numerical semigroup and let $x \in S$. Then $S \backslash\{x\}$ is a numerical semigroup if and only if $x \in \operatorname{msg}(S)$.

Proposition 2.8. If $S$ is a C-semigroup, then $\bar{S}=S \backslash\{M(S)\}$ is a symmetric numerical semigroup with $\mathrm{m}(\bar{S})=\mathrm{m}(S)$ and $\mathrm{F}(\bar{S})=\mathrm{F}(S)+\mathrm{m}(S)$.

Proof. Using Theorem 2.5 and Lemma 2.6, we obtain that $M(S)=\mathrm{F}(S)+\mathrm{m}(S)$. By Lemma 2.7, we get that $\bar{S}$ is numerical semigroup with $\mathrm{m}(\bar{S})=\mathrm{m}(S)$ and $\mathrm{F}(\bar{S})=\mathrm{F}(S)+$ $\mathrm{m}(S)$.

In order to prove that $\bar{S}$ is symmetric, we will see that if $x \in \mathbb{N} \backslash \bar{S}$ then $\mathrm{F}(\bar{S})-x \in \bar{S}$. If $x \in \mathbb{N} \backslash \bar{S}$, then either $x \in \mathbb{N} \backslash S$ or $x=M(S)$. We distinguish two cases. 
- If $x=M(S)$, then $\mathrm{F}(\bar{S})-x=M(S)-M(S)=0 \in \bar{S}$

- If $x \in \mathbb{N} \backslash S$, then $\mathrm{F}(S)+\mathrm{m}(S)-x \in S$ and so $\mathrm{F}(S)+\mathrm{m}(S)-x \in \bar{S}$ or $\mathrm{F}(S)+$ $\mathrm{m}(S)-x=M(S)$. If $\mathrm{F}(S)+\mathrm{m}(S)-x=M(S)$, then $x=0$, which contradicts $x \in \mathbb{N} \backslash \bar{S}$. Hence $\mathrm{F}(\bar{S})-x \in \bar{S}$ and thus $\bar{S}$ is symmetric.

The next result it easy to prove.

Lemma 2.9. If $S$ is a numerical semigroup such that $S \neq \mathbb{N}$, then $S \cup\{\mathrm{F}(S)\}$ is again a numerical semigroup.

The following result can be deduce of [9, Lemmas 1.2 and 1.3].

Lemma 2.10. Let $S$ be a symmetric numerical semigroup such that $S \notin\{\mathbb{N},\langle 2,3\rangle\}$ and let $\bar{S}=S \cup\{\mathrm{F}(S)\}$. Then $\bar{S}$ is a numerical semigroup with $\mathrm{F}(\bar{S})=\mathrm{F}(S)-\mathrm{m}(S)$ and $\mathrm{m}(\bar{S})=\mathrm{m}(S)$.

Proposition 2.11. If $S$ is a symmetric numerical semigroup such that $S \neq \mathbb{N}$, then $\bar{S}=S \cup\{\mathrm{F}(S)\}$ is a positioned numerical semigroup.

Proof. We distinguish two cases.

- If $S=\langle 2,3\rangle$ then $\bar{S}=\mathbb{N}$ and thus $\bar{S}$ is a positioned numerical semigroup.

- If $S \neq\langle 2,3\rangle$, then by Lemma 2.10 , we have that $\mathrm{m}(\bar{S})=\mathrm{m}(S)$ and $\mathrm{F}(\bar{S})+\mathrm{m}(\bar{S})=$ $\mathrm{F}(S)$. Whence, if $x \in \mathbb{N} \backslash \bar{S}$, then $x \in \mathbb{N} \backslash S$ and so $\mathrm{F}(S)-x \in S$. Consequently, $\mathrm{F}(\bar{S})+\mathrm{m}(\bar{S})-x \in \bar{S}$ and thus $\bar{S}$ is a positioned numerical semigroup.

A numerical semigroup $S$ is called a UESYsemigroups if there exists $S^{\prime}$ symmetric such that $S=S^{\prime} \cup\left\{\mathrm{F}\left(S^{\prime}\right)\right\}$.

Proposition 2.12. [9, Theorem 1.8] Let $S$ be a numerical semigroup such that $S \neq \mathbb{N}$. The following conditions are equivalent.

(1) $S$ is a UESYsemigroup.

(2) $\mathrm{F}(S)+\mathrm{m}(S) \in \operatorname{msg}(S)$ and $\mathrm{g}(S)=\frac{\mathrm{F}(S)+\mathrm{m}(S)-1}{2}$.

Theorem 2.13. Let $S$ be a numerical semigroup such that $S \neq \mathbb{N}$. Then $S$ is a $C$ semigroup if and only if $S$ is a UESYsemigroup.

Proof. Necessity. If $S$ is a $C$-semigroup, then $\mathrm{g}(S)=\frac{\mathrm{F}(S)+\mathrm{m}(S)-1}{2}$. By Theorem 2.5, we have that $\mathrm{F}(S)+\mathrm{m}(S) \in \operatorname{msg}(S)$. Hence, by applying Proposition 2.12 , we obtain that $S$ is a UESYsemigroup.

Sufficiency. If $S$ is a UESYsemigroup then there exist a symmetric numerical semigroup $S^{\prime}$ such that $S=S^{\prime} \cup\left\{\mathrm{F}\left(S^{\prime}\right)\right\}$ with $S^{\prime} \notin\{\langle 2,3\rangle, \mathbb{N}\}$. By Propositions 2.11 and 2.12, we have that $S$ is a positioned semigroup and $\mathrm{g}(S)=\frac{\mathrm{F}(S)+\mathrm{m}(S)-1}{2}$. Whence $S$ is a $C$-semigroup.

By using Theorem 2.13 and [9, Corollary 1.9 and Theorem 1.14] we obtain the following result.

Proposition 2.14. Let $S$ be a C-semigroup. Then the following conditions hold.

(1) $\mathrm{F}(S)=M(S)-\mathrm{m}(S)$.

(2) $\mathrm{g}(S)=\frac{M(S)-1}{2}$.

(3) $\operatorname{PF}(S)=\left\{{ }^{2} M(S)-x \mid x \in \operatorname{msg}(S)\right.$ and $\left.x \neq M(S)\right\}$ and $\mathrm{t}(S)=\mathrm{e}(S)-1$.

Note that $\mathbb{N}$ is the unique numerical semigroup with embedding dimension one which is not $C$-semigroup and so there are no $C$-semigroups with embedding dimension one.

In [11] it is proved that, if $S$ is a numerical semigroup with e $(S)=2$, then $S$ is symmetric and $\mathrm{g}(S)=\frac{\mathrm{F}(S)+1}{2}$. We deduce that if $S$ is a $C$-semigroup with e $(S)=2$, then this forces 
$\mathrm{m}(S)=2$. Since all symmetric numerical semigroups are positioned (see [2]) we obtain the next result.

Proposition 2.15. The set of $C$-semigroups with embedding dimension two is equal to $\{\langle 2,2 k+1\rangle \mid k \in \mathbb{N} \backslash\{0\}\}$.

It is well known that if $S$ is a numerical semigroup, then $\mathrm{e}(S) \leq \mathrm{m}(S)$ and $\mathrm{t}(S) \leq m-1$ (see [11]). The next aim, in this section, is to show that for given $m$ and $e$ integers (resp. $m$ and $t$ integers) such that $3 \leq e \leq m$ (resp. $2 \leq t \leq m-1$ ) there exists a $C$-semigroup with multiplicity $m$ and embedding dimension $e$ (resp. with multiplicity $m$ and type $t$ ).

The next lemmas are known and they are in [9].

Lemma 2.16. [9, Lemma 1.13] Let $S$ be a symmetric numerical semigroup with $\mathrm{m}(S) \geq 3$. Then $\mathrm{e}(S \cup\{\mathrm{F}(S)\})=\mathrm{e}(S)+1$ and $\mathrm{t}(S \cup\{\mathrm{F}(S)\})=\mathrm{e}(S)$.

Lemma 2.17. [9, Lemma 1.16] Let $m$ and e be integers such that $2 \leq e \leq m-1$. Then there exists a symmetric numerical semigroup $S$ with $\mathrm{m}(S)=m$ and $\mathrm{e}(S)=e$.

Proposition 2.18. Let $m$ and $e$ be integers such that $3 \leq e \leq m$. Then there exists a $C$-semigroup $S$ with $\mathrm{m}(S)=m$ and $\mathrm{e}(S)=e$.

Proof. By Lemma 2.17, there exists a symmetric numerical semigroup $S^{\prime}$ with $\mathrm{m}\left(S^{\prime}\right)=m$ and $\mathrm{e}\left(S^{\prime}\right)=e-1$. Suppose that $S=S^{\prime} \cup\left\{\mathrm{F}\left(S^{\prime}\right)\right\}$. Then $S$ is a UESYsemigroup and, by Theorem 2.13, is a $C$-semigroup. Further, by Lemmas 2.10 and 2.16, we obtain that $\mathrm{m}(S)=m$ and $\mathrm{e}(S)=e$.

Remark 2.19. Observe that $S$ is a symmetric numerical semigroup if and only if $\mathrm{t}(S)=1$. Besides, by the previous comment to Proposition 2.15 about the multiplicity, we obtain that the set of $C$-semigroups with type one is equal to $\{\langle 2,2 k+1\rangle \mid k \in \mathbb{N} \backslash\{0\}\}$.

Proposition 2.20. Let $m$ and $t$ be integers such that $2 \leq t \leq m-1$. Then there exists a $C$-semigroup $S$ with $\mathrm{m}(S)=m$ and $\mathrm{t}(S)=t$.

Proof. By Lemma 2.18, there exists a $C$-semigroup $S$ with $\mathrm{m}(S)=m$ and $\mathrm{e}(S)=t+1$. By applying Proposition 2.14, $\mathrm{t}(S)=t$.

Example 2.21. The numerical semigroup $S^{\prime}=\langle 7,11\rangle$ is a symmetric numerical semigroup with $\mathrm{F}\left(S^{\prime}\right)=59$. Hence $S^{\prime} \cup\left\{\mathrm{F}\left(S^{\prime}\right)\right\}=\langle 7,11,59\rangle$ is a UESYsemigroup and, by Theorem 2.13 , is a $C$-semigroup. Using Proposition 2.14 , we have that $\mathrm{F}\left(S^{\prime} \cup\left\{\mathrm{F}\left(S^{\prime}\right)\right\}\right)=59-7=52$, $\mathrm{g}\left(S^{\prime} \cup\left\{\mathrm{F}\left(S^{\prime}\right)\right\}\right)=\frac{59-1}{2}=29, \operatorname{PF}\left(S^{\prime} \cup\left\{\mathrm{F}\left(S^{\prime}\right)\right\}\right)=\{59-7,59-11\}=\{52,48\}$ and $\mathrm{t}\left(S^{\prime} \cup\left\{\mathrm{F}\left(S^{\prime}\right)\right\}\right)=2$.

\section{D-semigroups}

Recall that a $D$-semigroup is a positioned numerical semigroup $S$ with $\mathrm{g}(S)=\frac{\mathrm{F}(S)+\mathrm{m}(S)-2}{2}$. Our aim in this section is to characterize these types of numerical semigroups.

Example 3.1. Let us see that $S=\langle 6,7,11\rangle$ is a $D$-semigroup.

In fact $S=\{0,6,7,11,12,13,14,17, \rightarrow\}$ and thus $\mathrm{F}(S)=16, \mathrm{~m}(S)=6$, $\mathbb{N} \backslash S=\{1,2,3,4,5,8,9,10,15,16\}$ and $\mathrm{g}(S)=10$.

Moreover $\{\mathrm{F}(S)+\mathrm{m}(S)-x \mid x \in \mathbb{N} \backslash S\}=\{21,20,19,18,17,14,13,12,7,6\} \subseteq S$ and so $S$ is positioned. Since $\mathrm{g}(S)=\frac{\mathrm{F}(S)+\mathrm{m}(S)-2}{2}$, we obtain that $S$ is a $D$-semigroup

Lemma 3.2. If $S$ is a positioned numerical semigroup and $\mathrm{F}(S)+\mathrm{m}(S)$ is an even number, then $\frac{\mathrm{F}(S)+\mathrm{m}(S)}{2} \in S$.

Proof. Since $S$ is positioned, if $\frac{\mathrm{F}(S)+\mathrm{m}(S)}{2} \in \mathbb{N} \backslash S$ then $\mathrm{F}(S)+\mathrm{m}(S)-\frac{\mathrm{F}(S)+\mathrm{m}(S)}{2} \in S$. Hence $\frac{\mathrm{F}(S)+\mathrm{m}(S)}{2} \in S$, a contradiction. 
Lemma 3.3. Let $S$ be a positioned numerical semigroup. Then $S$ is a D-semigroup if and only if $\mathrm{g}(S)=\mathrm{q}(S)-1$.

Proof. If $S$ be a positioned numerical semigroup, then $\mathrm{g}(S)+\mathrm{q}(S)=\mathrm{F}(S)+\mathrm{m}(S)-1$. Hence, we obtain that $S$ is a $D$-semigroup (i.e. $2 \mathrm{~g}(S)=\mathrm{F}(S)+\mathrm{m}(S)-2$ ) if and only if $\mathrm{g}(S)=\mathrm{q}(S)-1$.

Lemma 3.4. If $S$ is a D-semigroup, then $\frac{\mathrm{F}(S)+\mathrm{m}(S)}{2} \in \operatorname{msg}(S)$ and $\mathrm{F}(S)+m(S)$ has unique expression in $S$.

Proof. By Lemma 3.2, we have that $\frac{\mathrm{F}(S)+\mathrm{m}(S)}{2} \in S$. Since $S$ is a $D$-semigroup, then $S \neq \mathbb{N}$ and so $\frac{\mathrm{F}(S)+\mathrm{m}(S)}{2} \neq 0$. If $\frac{\mathrm{F}(S)+\mathrm{m}(S)}{2} \notin \mathrm{msg}(S)$, then there exist $x, y \in S \backslash\{0\}$ such that $\frac{\mathrm{F}(S)+\mathrm{m}(S)}{2}=x+y$. Hence $\mathrm{F}(S)+\mathrm{m}(S)=2 x+2 y=x+(x+2 y)=(2 x+y)+y$. Now, by using Lemma 2.2 , we obtain that $\{x, 2 x, 2 x+y\} \subseteq Q(S)$ and $\{x, 2 x, 2 x+y\} \cap \phi(\mathbb{N} \backslash S)=\emptyset$ and thus $\mathrm{q}(S) \geq \mathrm{g}(S)+3$.

If $\mathrm{F}(S)+\mathrm{m}(S)$ has not unique expression in $S$, then there exist $x, y \in S \backslash\{0\}$ such that $\mathrm{F}(S)+\mathrm{m}(S)=x+y$ and $\frac{\mathrm{F}(S)+\mathrm{m}(S)}{2} \notin\{x, y\}$. By Lemma 2.2 again, we get that $\mathrm{q}(S) \geq \mathrm{g}(S)+2$.

In both cases, we have that if $\frac{\mathrm{F}(S)+\mathrm{m}(S)}{2} \notin \mathrm{msg}(S)$ or if $\mathrm{F}(S)+\mathrm{m}(S)$ does not have unique expression in $S$, then $\mathrm{g}(S) \neq \mathrm{q}(S)-1$. But this contradicts Lemma 3.3, since $S$ is a $D$-semigroup.

Theorem 3.5. Let $S$ be a positioned numerical semigroup. Then $S$ is a D-semigroup if and only if $\frac{\mathrm{F}(S)+\mathrm{m}(S)}{2} \in \operatorname{msg}(S)$ and $\mathrm{F}(S)+\mathrm{m}(S)$ has unique expression in $S$.

Proof. Necessity. This is an immediate consequence of the Lemma 3.4.

Sufficiency. From the hypothesis, we deduce that $\frac{\mathrm{F}(S)+\mathrm{m}(S)}{2}$ is the unique element in $Q(S) \backslash \operatorname{Im}(\phi)$ (in view of of Lemma 2.2) and thus $\mathrm{g}(S)=\mathrm{q}(S)-1$. By using Lemma 3.3, we have that $S$ is a $D$-semigroup.

Lemma 3.6. If $S$ is a D-semigroup, then

$$
\bar{S}=S \backslash\left\{\frac{\mathrm{F}(S)+\mathrm{m}(S)}{2}, \mathrm{~F}(S)+\mathrm{m}(S)\right\}
$$

is a numerical semigroup.

Proof. As by Theorem 3.5, we have that $\frac{\mathrm{F}(S)+\mathrm{m}(S)}{2} \in \operatorname{msg}(S)$, then, by applying Lemma $2.7, S^{\prime}=S \backslash\left\{\frac{\mathrm{F}(S)+\mathrm{m}(S)}{2}\right\}$ is a numerical semigroup. Using again Theorem $3.5, \mathrm{~F}(S)+\mathrm{m}(S)$ has unique expression in $S$. We can deduce that $\mathrm{F}(S)+\mathrm{m}(S) \in \operatorname{msg}\left(S^{\prime}\right)$ and in the same way $\bar{S}=S^{\prime} \backslash\{\mathrm{F}(S)+\mathrm{m}(S)\}$ is a numerical semigroup.

Proposition 3.7. If $S$ is a D-semigroup, then

$$
\bar{S}=S \backslash\left\{\frac{\mathrm{F}(S)+\mathrm{m}(S)}{2}, \mathrm{~F}(S)+\mathrm{m}(S)\right\}
$$

is a pseudosymmetric numerical semigroup with $2 \mathrm{~m}(\bar{S})<\mathrm{F}(\bar{S})$.

Proof. Obviously $\mathrm{F}(\bar{S})=\mathrm{F}(S)+\mathrm{m}(S)$. We need to show that $\bar{S}$ is a pseudosymmetric numerical semigroup (i.e. $x \in \mathbb{Z} \backslash \bar{S}$ implies that either $\mathrm{F}(\bar{S})-x \in \bar{S}$ or $\left.x=\frac{\mathrm{F}(\bar{S})}{2}\right)$.

We have that if $x \in \mathbb{N} \backslash \bar{S}$ then either $x \in \mathbb{N} \backslash S$ or $x=\frac{\mathrm{F}(S)+\mathrm{m}(S)}{2}$ or $x=\mathrm{F}(S)+\mathrm{m}(S)$. We distinguish three cases.

- If $x \in \mathbb{N} \backslash S$ then $\mathrm{F}(S)+\mathrm{m}(S)-x \in S$ and so $\mathrm{F}(\bar{S})-x \in S$. Hence either $\mathrm{F}(\bar{S})-x \in \bar{S}$ or $\mathrm{F}(\bar{S})-x=\frac{F(\bar{S})}{2}$ or $\mathrm{F}(\bar{S})-x=\mathrm{F}(\bar{S})$. As the last two cases are not possible, we get that $\mathrm{F}(\bar{S})-x \in \bar{S}$. 
- If $x=\frac{\mathrm{F}(S)+\mathrm{m}(S)}{2}$, then $2 x=\mathrm{F}(\bar{S})$, contradicting that $2 x \neq \mathrm{F}(\bar{S})$.

- If $x=\mathrm{F}(S)+\mathrm{m}(S)$, then $x=\mathrm{F}(\bar{S})$ and $\mathrm{F}(\bar{S})-x=\mathrm{F}(\bar{S})-\mathrm{F}(\bar{S})=0 \in \bar{S}$.

Since $\mathrm{F}(S)+\mathrm{m}(S)$ is even, then $\mathrm{F}(S) \neq \mathrm{m}(S)-1$ and so $\mathrm{F}(S)>\mathrm{m}(S)$. Therefore $\mathrm{m}(S)=\mathrm{m}(\bar{S})$ and $\mathrm{F}(\bar{S})>2 \mathrm{~m}(\bar{S})$.

As a consequence of [8, Lemmas 31 and 32$]$ we obtain the following.

Lemma 3.8. Let $S$ be a pseudosymmetric numerical semigroup with $\mathrm{F}(S)>2 \mathrm{~m}(S)$. Then $\bar{S}=S \cup\left\{\frac{\mathrm{F}(S)}{2}, \mathrm{~F}(S)\right\}$ is a numerical semigroup, $\mathrm{m}(\bar{S})=\mathrm{m}(S)$ and $\mathrm{F}(\bar{S})=\mathrm{F}(S)-\mathrm{m}(S)$

Proposition 3.9. Let $S$ be a pseudosymmetric numerical semigroup with $\mathrm{F}(S)>2 \mathrm{~m}(S)$. Then $\bar{S}=S \cup\left\{\frac{\mathrm{F}(S)}{2}, \mathrm{~F}(S)\right\}$ is a positioned numerical semigroup.

Proof. From Lemma 3.8 we obtain that $\bar{S}$ is a numerical semigroup, $\mathrm{m}(\bar{S})=\mathrm{m}(S)$ and $\mathrm{F}(\bar{S})=\mathrm{F}(S)-\mathrm{m}(S)$. We need to see that $\bar{S}$ is positioned. If $x \in \mathbb{N} \backslash \bar{S}$ then $x \in \mathbb{N} \backslash S$ and $x \neq \frac{\mathrm{F}(S)}{2}$. Since $S$ is pseudosymmetric, we obtain that $\mathrm{F}(S)-x \in S \subseteq \bar{S}$. Hence $\mathrm{F}(\bar{S})+\mathrm{m}(\bar{S})-x \in \bar{S}$ and so $\bar{S}$ is positioned.

A numerical semigroup $S$ is called PEPSY-semigroup if there exist $S^{\prime}$ pseudosymmetric numerical semigroup such that $S=S^{\prime} \cup\left\{F\left(S^{\prime}\right), \frac{\mathrm{F}\left(S^{\prime}\right)}{2}\right\}$.

A PEPSY-semigroup that is not a half-line is called PEPSYNHL-semigroup.

From this definitions we have the following results of [8].

Lemma 3.10. [8, Proposition 30] A numerical semigroup $S$ is a PEPSY-semigroup if and only if one of the following conditions holds:

(1) $S$ is half-line.

(2) there exists a pseudo-symmetric numerical semigroup $S^{\prime}$ with $F\left(S^{\prime}\right)>2 \mathrm{~m}\left(S^{\prime}\right)$ and $S=S^{\prime} \cup\left\{F\left(S^{\prime}\right), \frac{\mathrm{F}\left(S^{\prime}\right)}{2}\right\}$.

Lemma 3.11. [8, Theorem 33] Let $S$ not be half-line. The following conditions are equivalent:

(1) $S$ is a PEPSY-semigroup,

(2) $\frac{\mathrm{F}(S)+\mathrm{m}(S)}{2} \in \operatorname{msg}(S), \mathrm{F}(S)+\mathrm{m}(S)$ has unique expression in $S$ and $\mathrm{g}(S)=\frac{\mathrm{F}(S)+\mathrm{m}(S)-2}{2}$.

Theorem 3.12. A semigroup $S$ is a D-semigroup if and only if $S$ is a PEPSYNHLsemigroup.

Proof. Necessity. If $S$ is a $D$-semigroup, then we have that $\mathrm{F}(S)+\mathrm{m}(S)$ is even and thus $S$ is not half-line. Besides, we have that $\mathrm{g}(S)=\frac{\mathrm{F}(S)+\mathrm{m}(S)-2}{2}$ and, by Theorem 3.5, $\frac{\mathrm{F}(S)+\mathrm{m}(S)}{2} \in \operatorname{msg}(S)$ and $\mathrm{F}(S)+\mathrm{m}(S)$ has unique expression in $S$. Hence we conclude, by Lemma 3.11, that $S$ is a PEPSYNHL-semigroup.

Sufficiency. Suppose that $S$ is a PEPSYNHL-semigroup. By using Lemma 3.10, there exists a pseudo-symmetric numerical semigroup $S^{\prime}$ with $F\left(S^{\prime}\right)>2 \mathrm{~m}\left(S^{\prime}\right)$ and $S=S^{\prime} \cup$ $\left\{F\left(S^{\prime}\right), \frac{\mathrm{F}\left(S^{\prime}\right)}{2}\right\}$. By Lemma 3.8 and Proposition 3.9, we get that $S$ is positioned and $\mathrm{m}\left(S^{\prime}\right)=\mathrm{m}(S)$ and $\mathrm{F}(S)=\mathrm{F}\left(S^{\prime}\right)-\mathrm{m}\left(S^{\prime}\right)$. As $S^{\prime}$ is pseudo-symmetric then $\mathrm{g}\left(S^{\prime}\right)=\frac{\mathrm{F}\left(S^{\prime}\right)+2}{2}$ and $\mathrm{g}(S)=\mathrm{g}\left(S^{\prime}\right)-2$. Therefore, we can conclude that $\mathrm{g}(S)=\frac{\mathrm{F}\left(S^{\prime}\right)+2}{2}-2=\frac{\mathrm{F}(S)+\mathrm{m}(S)-2}{2}$ and so $S$ is a $D$-semigroup.

As a consequence of Lemma 3.10 and Theorem 3.12 we obtain the following result.

Corollary 3.13. The set of all D-semigroups is equal to

$$
\left\{S^{\prime} \cup\left\{\mathrm{F}\left(S^{\prime}\right), \frac{\mathrm{F}\left(S^{\prime}\right)}{2}\right\} \mid S^{\prime} \text { pseudo-symmetric semigroup with } \mathrm{F}\left(S^{\prime}\right)>2 \mathrm{~m}\left(S^{\prime}\right)\right\} \text {. }
$$


The next results give us formulas for the Frobenius number and pseudo-Frobenius numbers of a $D$-semigroup. The first (resp. second) is a consequence of Theorem 3.12 and [8, Corollary 34] (resp. Theorem 3.12 and [8, Lemma 35 and Theorem 38]).

Proposition 3.14. If $S$ is a D-semigroup, then $\mathrm{F}(S) \leq 2 M(S)-\mathrm{m}(S)$. Moreover, $\mathrm{F}(S)=2 x-\mathrm{m}(S)$ for some $x \in \operatorname{msg}(S)$ such that $2 x>M(S)$ and $2 x$ has unique expression in $S$.

Proposition 3.15. If $S$ is a D-semigroup, then $\mathrm{t}(S)=\mathrm{e}(S)-1$. Moreover, $\operatorname{PF}(S)=$ $\left\{\mathrm{F}(S)+\mathrm{m}(S)-x \mid x \in \operatorname{msg}(S)\right.$ and $\left.x \neq \frac{\mathrm{F}(S)+\mathrm{m}(S)}{2}\right\}$.

\section{The algorithm}

Given positive integers $m$ and $F$, we denote by

$$
\begin{aligned}
P(m, F)= & \{S \mid S \text { is a positioned semigroup with } \mathrm{m}(S)=m \text {, and } \mathrm{F}(S)=F\}, \\
& \text { and } \mathfrak{P}(m, F)=\left\{S \in P(m, F) \mid \mathrm{g}(S)=\left\lfloor\frac{F+m-1}{2}\right\rfloor\right\} .
\end{aligned}
$$

The aim of this section is to show how to construct an algorithm to compute all elements in $\mathfrak{P}(m, F)$.

Clearly, if $C(m, F)=\{S \in P(m, F) \mid S$ is a $C$-semigroup $\}$ and

$D(m, F)=\{S \in P(m, F) \mid S$ is a $D$-semigroup $\}$, then

$$
\mathfrak{P}(m, F)= \begin{cases}C(m, F) & \text { if } F+m \text { is odd } \\ D(m, F) & \text { if } F+m \text { is even } .\end{cases}
$$

\subsection{Case $m+F$ odd}

Since $\mathrm{g}(\mathbb{N})=0$ we have that $\mathbb{N}$ is not a $C$-semigroup. By Remark 2.19, we deduce that the whole set of $C$-semigroups with $\mathrm{m}(S)=2$ is equal to $\{\langle 2,2 k+1\rangle \mid k \in \mathbb{N} \backslash\{0\}\}$. Since $\mathrm{F}(\langle 2,2 k+1\rangle)=2 k-1$, then $F$ is an odd integer if and only if $C(2, F) \neq \emptyset$. Therefore, we can conclude that $C(2, F)=\{\langle 2, F+2\rangle\}$.

From now on we assume that $m \geq 3$. Clearly, if $S$ is a numerical semigroup and $\mathrm{m}(S)=m$, then $\mathrm{F}(S) \geq m-1$. If $\mathrm{F}(S)=m-1$ then $S=\Delta(m)$ is a half-line, and by Proposition 1, it is $C$-semigroup. Therefore, we have that $C(m, m-1)=\{\Delta(m)\}$.

So let us assume that $3 \leq m<F$ and $F+m$ is odd. From Lemma 2.10 and Theorem 2.13 we deduce the next result.

Proposition 4.1. With the above notation, we have that

$$
\begin{aligned}
C(m, F) & =\{S \cup\{\mathrm{F}(S)\} \mid S \text { is a symmetric } \\
& \text { numerical semigroup, } \mathrm{m}(S)=m \text { and } \mathrm{F}(S)=F+m\} .
\end{aligned}
$$

We denote by $\mathfrak{I}(m, F)$ the set of all irreducible numerical semigroups with multiplicity $m$ and Frobenius number $F$. Recall that a numerical semigroup is symmetric (respectively pseudo-symmetric) if it is irreducible with Frobenius number odd (respectively even).

Lemma 4.2. [4, Proposition 13] Let $m$ and $F$ be integers such that $F \geq 3$. Then $\Im(m, F) \neq \varnothing$ if and only if $m \leq \frac{F+2}{2}$ and $m \nmid F$.

As a consequence of Proposition 4.1 and Lemma 4.2 we have the following.

Proposition 4.3. With the above notation, we have that $C(m, F) \neq \varnothing$ if and only if $m \nmid F$. 
Now, we are able to give an algorithm to compute the whole set $C(m, F)$.

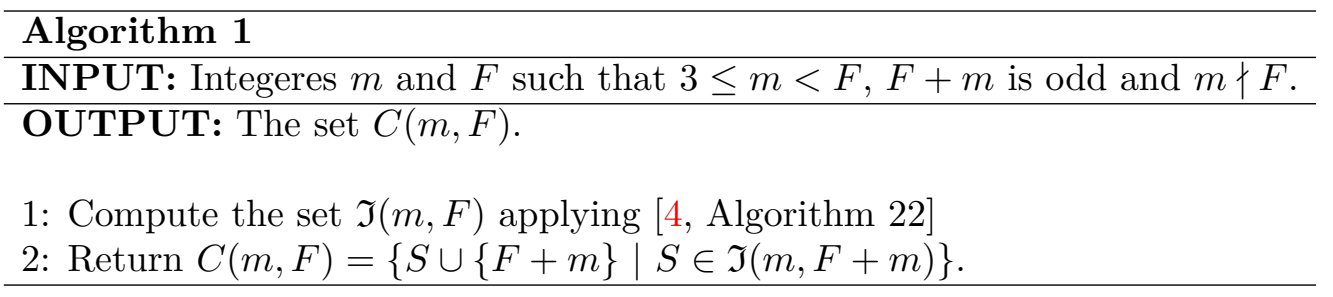

Example 4.4. Let us compute the whole set $C(6,13)$.

(1) Compute the set $\mathfrak{I}(6,19)$ applying $[4$, Algorithm 22]. We start by the root of the tree $\langle 6,10,11,14,15\rangle$ and we obtain that $\mathfrak{I}(6,19)=\{\langle 6,10,11,14,15\rangle,\langle 6,8,10,15,17\rangle,\langle 6,9,11,14,16\rangle,\langle 6,8,9\rangle\}$.

(2) $C(6,13)=\{\langle 6,10,11,14,15,19\rangle,\langle 6,8,10,15,17,19\rangle, 6,9,11,14,16,19\rangle,\langle 6,8,9,19\}$.

\subsection{Case $m+F$ even}

From previous results we have that there are no $D$-semigroups with multiplicity 1 and 2 . On the other hand, as a half-line is not a $D$-semigroup we obtain that if $S$ is a $D$-semigroup then $\mathrm{F}(S)>\mathrm{m}(S)$. So let us assume that $3 \leq m<F$ and $F+m$ is even.

As a consequence of Theorem 3.12 and Lemma 3.8 we have the following.

Proposition 4.5. With the above notation, we have that

$$
\begin{aligned}
& D(m, F)=\left\{S \cup\left\{F+m, \frac{F+m}{2}\right\} \mid S\right. \text { is a pseudosymmetric } \\
& \text { numerical semigroup, } \mathrm{m}(S)=m \text { and } \mathrm{F}(S)=F+m\} \text {. }
\end{aligned}
$$

From Lemma 4.2 and Proposition 4.5 we deduce the next result.

Proposition 4.6. With the above notation, we have that $D(m, F) \neq \varnothing$ if and only if $m \nmid F$.

We conclude by giving an algorithm that will allow us to compute the whole set $D(m, F)$

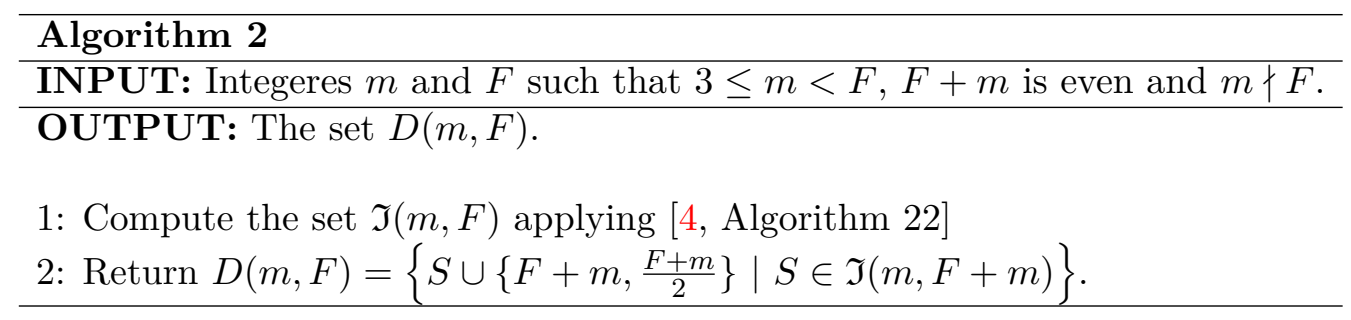

Acknowledgment. We thank the anonymous referees for their detailed suggestions and comments, which have greatly improved this article. 


\section{References}

[1] V. Barucci, D.E. Dobbs and M. Fontana, Maximality Properties in Numerical Semigroups and Applications to One-Dimensional Analytically Irreducible Local Domains, Memoirs of the Amer. Math. Soc. 598, 1997.

[2] M.B. Branco, M.C. Faria and J.C. Rosales, Positioned numerical semigroups, Quaest. Math. 44, 679-691, 2021.

[3] M.B. Branco, M.C. Faria and J.C. Rosales, Almost-positioned numerical semigroups, Results Math. 76, 1-14, 2021.

[4] M.B. Branco, I. Ojeda and J.C. Rosales, The set of numerical semigroups of a given multiplicity and Frobenius number, Port. Math. 78, 147-167, 2021.

[5] R. Fröberg, C. Gottlieb and R. Häggkvist, On numerical semigroups, Semigroup Forum 35, 63-83, 1987.

[6] E. Kunz, The value-semigroup of a one-dimensional Gorenstein ring, Proc. Amer. Math. Soc. 25, 748-751, 1973.

[7] J.C. Rosales, On symmetric numerical semigroups, J. Algebra 182, 422-434, 1996.

[8] J.C. Rosales, Adding or removing on element from a Pseudo-symmetric numerical semigroup, Boll. Unione Mat. Ital. 9, 681-696, 2006.

[9] J.C. Rosales, Numerical semigroups that differ from a Symmetric numerical semigroups in one element, Algebra Colloq. 15, 23-32, 2008.

[10] J.C. Rosales and M.B. Branco, Irreducible numerical semigroups, Pacific J. Math. 209, 131-143, 2003.

[11] J.C. Rosales and P.A. García-Sánchez, Numerical semigroups, Springer Science \& Business Media, 2009. 